\section{$\underset{\text { hommes }}{\text { \& migrations }}$}

\section{Hommes \& migrations}

Revue française de référence sur les dynamiques

migratoires

$1310 \mid 2015$

Fashion Mix

\title{
Habits et habitus en héritage
}

Les designers de la diaspora chinoise à New York

\section{Leyla Belkaïd}

\section{(2) OpenEdition}

1 Journals

\section{Édition électronique}

URL : http://journals.openedition.org/hommesmigrations/3159

DOI : 10.4000/hommesmigrations.3159

ISSN : 2262-3353

Éditeur

Musée national de l'histoire de l'immigration

\section{Édition imprimée}

Date de publication : 1 avril 2015

Pagination : 85-90

ISBN : 978-2-919040-31-5

ISSN : 1142-852X

\section{Référence électronique}

Leyla Belkaïd, « Habits et habitus en héritage », Hommes \& migrations [En ligne], 1310 | 2015, mis en ligne le 01 avril 2018, consulté le 20 avril 2019. URL : http://journals.openedition.org/

hommesmigrations/3159; DOI : 10.4000/hommesmigrations.3159 


\title{
HABITS ET HABITUS EN HÉRITAGE \\ LES DESIGNERS DE LA DIASPORA CHINOISE À NEW YORK
}

Par LEYLA BELKAÏD, designer et docteur en ethnologie, chercheur indépendant en anthropologie de la mode.

\begin{abstract}
À New York, dans le domaine du prêt-à-porter haut de gamme, les créateurs de mode sino-américains déjouent les assignations identitaires. S'ils véhiculent, emploient et redessinent certaines références culturelles liées à la Chine, leur travail est aux antipodes des stéréotypes véhiculés par la culture de masse. Dans leurs créations, point de dragons rouges et autres chapeaux pointus, mais des souvenirs de tenues, de froissements d'étoffe, d'attitudes du corps qui les nourrissent et qui rappellent que le vêtement est aussi un imaginaire que l'on porte sur soi.
\end{abstract}

Pour Stuart Hall, l'expérience diasporique se traduit par une conception identitaire qui n'évolue pas malgré la différence, mais avec la différence et à travers elle ${ }^{1}$. Mon étude s'appuie sur une enquête ethnographique menée aux États-Unis entre 2012 et 2013, avec l'objectif d'explorer l'impact de l'imagination diasporique sur la création de mode contemporaine. Elle examine, sous l'angle de la mnémonique des pratiques vestimentaires, les récits de vie de designers sino-américains indépendants ${ }^{2}$ nés en Asie orientale ${ }^{3}$ et actuellement actifs à New York ${ }^{4}$. Mon terrain s'est principalement organisé à Manhattan, dans le Garment District ${ }^{5}$, centre névralgique où les designers de mode profitent de la proximité des fournisseurs, des façonniers, des grands magasins et des agences de communication pour développer leurs marques de prêt-à-porter ${ }^{6}$. Aujourd'hui encore, malgré la sous- 
traitance et la délocalisation de la production textile ${ }^{7}$, les deux tiers de l'activité industrielle de mode de la mégapole se concentrent dans ce quartier historique, désormais rebaptisé "Fashion District".

\section{Les pionnières de la mode sino-américaine}

Bien que la création de mode qui émerge à New York pendant l'entre-deux-guerres se veuille démocratique ${ }^{8}$, en opposition à la haute couture parisienne, elle entretient la stratification de la société en termes de différences d'ethnies, de genres et de classes. Les couturières et les tailleurs chinois ont longtemps été écartés de l'histoire de la mode américaine, en dépit de leur participation précoce et décisive au développement de l'industrie de l'habillement. "Alors que les Chinois ont pleinement contribué à la construction de léconomie états-unienne, ils sont restés des étrangers exotiques et dangereux $x^{9}$." La transition du statut d'immigré, supposé réfractaire à toute assimilation ${ }^{10}$, à celui de citoyen américain a été et demeure laborieuse. Tandis que l'imaginaire états-unien identifie la femme asiatique à la blanchisseuse ou à la couturière assise derrière sa machine à coudre, l'ascension des designers de mode d'origine chinoise au sein de la Creative Class des années 1990 constitue un phénomène inédit, surtout que les premiers créateurs sinoaméricains à succès sont des femmes : Vera Wang, Anna Sui, Yeohlee Teng et Vivianne Tam. Pour l'opinion publique, l'American dream a permis de hisser la petite main chinoise au statut de designer entrepreneur. En réalité, aucune de ces nouvelles figures new-yorkaises de la mode ne rentrait dans le moule de l'immigrée asiatique ouvrière du textile. Toutes appartenaient à lélite sino-américaine avant de débuter leur activité, notamment Vera Wang, née à New York dans une famille aisée de l'Upper East Side, et Anna Sui, élevée par une mère artiste et un père architecte, tous deux formés à Paris. Les autres designers ont vécu en Asie et se sont déplacés à New York pour y suivre une formation supérieure en design de mode aux frais de leurs parents ${ }^{11}$.

Les pionnières chinoises du prêt-à-porter haut de gamme ne sont pas les filles des ouvrières qui s'épuisent à la tâche dans les sweatshops de Chinatown ou du Garment District. Néanmoins, leur apparition sur la scène de la mode new-yorkaise

3. Les designers investigués sont nés entre 1970 et 1980 dans différents pays d'Asie orientale (Chine, Taïwan, Thaïlande, Viêtnam, Malaisie). Ils se considèrent comme Chinois car leurs parents sont originaires de Chine et s'expriment en mandarin ou dans I'une des autres langues de ce pays. Arrivés aux États-Unis avant ou au début de l'adolescence, ils font partie de la 1,5 Generation qui désigne les enfants nés en Asie et immigrés en bas âge, aux côtés de leurs parents. Ils n'appartiennent ni à la première génération de migrants arrivés aux États-Unis à l'âge adulte, ni à la deuxième née en Amérique. 4. Le secteur d'activité de mes interlocuteurs se circonscrit à l'industrie de la mode américaine à forte valeur ajoutée créative ou prêt-à-porter de luxe (high-end design labels). Bien que certains produisent parallèlement une ligne masculine, le prêt-à-porter féminin (womenswear) constitue leur principal champ d'action. Le panel de designers ne se limite ni aux personnalités célèbres de la mode new-yorkaise, ni aux marques à fort potentiel financier, commercial et médiatique. Pour être représentatif de la population étudiée, il réunit les créateurs d'entreprises aux croissances hétérogènes, fondées à New York entre 2005 et 2010. L'échantillon final regroupe 7 designers (3 femmes et 4 hommes). Parmi eux, seuls 3 sont cités sous leur vrai nom, à savoir Wayne Lee (label Wayne), Phillip Lim (label 3.1 Phillip Lim) et Thomas Chen (label Emmanuelle), tandis que les 4 autres noms mentionnés dans le texte correspondent à des pseudonymes. Comme son entreprise réunit plus de 100 employés et possède 7 boutiques en propre, dont 5 en Asie orientale, Phillip Lim est le seul à bénéficier d'une réelle couverture médiatique au niveau national et international. Les trajectoires des autres designers investigués sont moins exceptionnelles dans la mesure où leur présence dans les médias et la diffusion de leurs collections progressent à un rythme plus lent, dans un marché particulièrement concurrentiel. 5. Le Garment District se situe entre la Cinquième et la Neuvième Avenue et entre la $34^{e}$ et la $40^{e}$ Rue. Mon terrain s'est étendu à d'autres zones de l'île de Manhattan où les designers ont choisi d'établir leurs studios, notamment Soho et Greenwich Village. 6. Stuart Hall, op. cit. 7. Une partie de la production de prêt-à-porter s'est déplacée vers le New Jersey à partir des années 1960, puis vers la Pennsylvanie et d'autres États, avant d'être délocalisée en Amérique latine et en Asie.

8. Rebecca Arnold, The American Look. Fashion, Sportswear and the Image of Women in 1930 s and 1940s New York, Londres et New York, I.B. Tauris, 2009. 9. Andrea Louie, Chineseness across Borders. Renegociating Chinese Identities in China and the United States, Durham et Londres, Duke University Press, 2004, p. 100. L'ensemble des extraits en langue anglaise cités dans ce texte ont été traduits par l'auteur. 10. Neil Gotanda, “Exclusion and inclusion: immigration and American orientalism”, in Evelyn Hu-DeHart (dir.), Across the Pacific. Asian Americans and Globalization, Philadelphie, Temple University Press, 1999, pp. 129-151; Lisa Lowe, Immigrant Acts. On Asian American Cultural Politics, Durham et Londres, Duke University Press, 2004 [1996], p. 5. 11. C'est le cas de Vivienne Tam (née à Guangzhou en 1957) qui passe son enfance à Hong Kong où elle étudie le fashion design, avant de parachever sa formation à Parsons The New School for Design de New York. Yeohlee Teng, issue d'une famille de commerçants chinois aisés vivant en Malaisie, s'installe également à New York pour étudier à Parsons, ainsi que Zang Toi, l'un des rares hommes de cette génération de designers d'origine asiatique, lui aussi né en Malaisie de parents chinois et formé à Parsons. 
ne passe pas inaperçue, car l'élite sino-américaine a longtemps privilégié les professions moins exposées, notamment dans la technologie et les sciences. S'affirmer dans un champ d'activité plus médiatisé reste une nouveauté, mais il ne s'agit pas d'un événement isolé puisque, à partir des années 1980, d'autres sujets d'origine chinoise commencent à être représentés dans des corps de métiers réputés inaccessibles aux membres de leur communauté, notamment la politique, le journalisme et les arts. L’analyse matérielle des collections de la première génération de designers de mode sino-américains montre que leurs productions s'inscrivent dans trois courants stylistiques : les styles conformes aux canons esthétiques américains de la haute société à dominante européenne (Vera Wang qui produit de luxueuses robes de mariée, et Zang Toi, créateur de robes du soir au style classique); les styles qui obéissent à la tendance Asian Chic $^{12}$, imprégnés de l'univers exotique supposé du designer (Vivienne $\mathrm{Tam}^{13}$ et, dans une moindre mesure, Anna Sui, chez qui les emprunts au répertoire orientalisant se dissolvent dans un univers punk); les styles minimalistes qui mettent en avant le travail de la géométrie et l'architecture du vêtement (Yeohlee Teng). La seconde génération de designers sinoaméricains qui lancent leurs marques de womenswear à partir des années 2000 provient souvent de familles immigrées modestes ${ }^{14}$, contrairement à leurs aînés. Les collections de prêt-à-porter qu'ils conçoivent à New York ne s'inscrivent dans aucune de ces trois orientations narratives et stylistiques. Létude comparative des techniques, des traits morphologico-esthétiques et des fonctions des objets qui les composent révèle l'existence d'emboîtements identitaires et mnémoniques plus complexes.

\section{Pratiques du vêtement vernaculaire}

L'analyse du style échafaudé par les jeunes designers de la 1,5 Generation passe par l'investigation de leur relation aux traditions vestimentaires chinoises et au corps en tant que matrice culturelle $e^{15}$. Bien qu'ils relatent l'insistance avec laquelle leurs parents les poussaient à suivre des cours du soir de mandarin ou à assimiler les valeurs confucéennes, mes interlocuteurs ne font jamais état d'une éventuelle contrainte liée au port du costume vernaculaire. Celui-ci se limite aux cérémonies familiales ou religieuses et ne concerne guère la sphère publique, en dehors de la communauté. La plupart du temps, c'est en rapport avec les pratiques des membres de leurs familles respectives, autant qu'avec leurs propres expériences corporelles, émotives et sensorielles, que les designers de mode mentionnent l'existence d'éléments vestimentaires chinois dans leur environnement intime.

L'évocation du vêtement traditionnel se rapporte de manière récurrente aux habits, aux postures corporelles quotidiennes, aux gestuelles et aux habitudes des mères, des grands-mères et des tantes, sujets qui véhiculent la culture chinoise à l'intérieur de la sphère familiale ${ }^{16}$. Le souvenir de la corporalité partagée ${ }^{17}$ à travers l'expérience du vêtement rattaché aux rituels familiaux semble avoir laissé une empreinte durable sur les subjectivités des designers. Corrélativement, la mémoire de ces derniers fait autant appel aux formes de ce vêtement qu'aux techniques du $\operatorname{corps}^{18}$ et aux conduites 
sensori-motrices $^{19}$ qui lui sont associées. Lisa (pseudonyme) compare les larges pantalons droits et les vestes boutonnées non ajustées au corps qui apparaissent sur les photos de famille quelle a conservées à des uniformes unisexes ${ }^{20}$. Elle affirme que les attitudes vestimentaires de ses proches ont influencé l'esthétique de ses collections parce qu'elle tend à privilégier les

L'évocation du vêtement traditionnel se rapporte de manière récurrente aux habits,

aux postures corporelles quotidiennes, aux gestuelles et aux habitudes des mères, des grands-mères et des tantes, sujets qui véhiculent la culture chinoise à l'intérieur de la sphère familiale. vêtements androgynes, les formes droites, l'ampleur des volumes dans l'espace, les couleurs sombres, ainsi que les différentes tonalités de blanc. La grand-mère de Peter (pseudonyme) continuait à porter de larges tuniques avec des pantalons et des vestes à l'ancienne, longtemps après son arrivée aux États-Unis. Il la trouvait bien mise et observait le grand soin qu'elle apportait à ses vêtements nettoyés soigneusement tous les jours, comme un soldat prenant soin de son uniforme. Le designer est persuadé qu'il y a en lui quelque chose qui le pousse à interpréter cette culture du vêtement à partir d'un lexique contemporain.

Les souvenirs qui refont surface au fil des récits décrivent d'autre part les sensations liées aux vêtements d'intérieur et de nuit. Cousus par les grands-mères ou par les mères, les pyjamas fluides marquent les sensations tactiles des enfants et leur sensibilité à la qualité de la matière textile. L'analyse technnologique des traits singularisants de quelques centaines de pièces récentes et d'archives corrobore l'usage récurrent de textiles soyeux souples, en particulier le crêpe de soie, dans les collections diffusées par la plupart de mes interlocuteurs. Elle montre aussi la présence d'une proportion élevée de vêtements au volume confortable et à la surface plane, non ajustée au corps.

Les conduites corporelles, sensorielles et affectives mémorisées par les designers se rapportent à des typologies particulière d'habits, d'espaces, d'actions et de séquences de vie. Leur mémoire revêt un caractère disloqué et parcellaire. Ted (pseudonyme) parvient, par exemple, à décrire le détail d'une broderie chinoise à motif animalier sur la robe qipao ${ }^{21}$ que portait sa mère à l'occasion d'un mariage, il y a trente ans, mais fait l'impasse sur la tenue vestimentaire avec laquelle il pratiquait les arts martiaux pendant ses années d'adolescence. Wayne Lee se remémore les trois vêtements qu'elle portait lors de sa périlleuse traversée du Pacifique à lâge de 6 ans $^{22}$, mais ne sait plus si elle a un jour porté le qipao. Pour les sujets diasporiques, "la relation au passé est toujours brisée par la guerre, l'emploi et le déplacement. La culture asiaticoaméricaine 'se souvient ${ }^{23}$ ' du passé dans et à travers la fragmentation, la perte, et la dispersion qui constitue le passé24".

Enfin, il ressort des entretiens que le souvenir du vêtement traditionnel chinois ne constitue pas un élément primordial du vécu familial. Ted fait d'ailleurs état du contraste entre son expérience personnelle et les attentes des journalistes de mode: "Je n'ai pas grandi dans un décor de Cité impériale, au milieu des mandarins en costume et tout le bazar chinois (Chinese stuff)... Pourtant, les journalistes de mode attendaient des vestes brodées de dragons, des robes bariolées, des broderies sur soie rouge, des chapeaux pointus..., c'est ce quills auraient voulu voir dans les collections des designers chinois. Un peu comme en Europe... il y a toujours eu cette attraction pour l'exotisme dans la mode. Ils attendaient des références

\footnotetext{
19. Jean-Pierre Warnier, Construire la culture matérielle. L'homme qui pensait avec ses doigts, Paris, PUF, 1999. 20. En Chine, l'effacement des différences vestimentaires entre les sexes s'accentue pendant la Révolution culturelle (1967-1976). Le rejet des modes occidentales et l'idéalisation de l'uniforme se matérialisent par l'image de la femme révolutionnaire et androgyne qui porte des vestes boutonnées jusqu'au cou et des pantalons droits en coton, dans des teintes sobres qui vont du kaki au bleu foncé. L’iconographie maoïste met également en avant l'idéal de la paysanne active, habillée en shanku avec pantalon large et veste de coupe rudimentaire.

21. La robe longue de type qipao (cheongsam en cantonais) s'apparente initialement à l'archétype masculin chinois appelé changpao, porté à l'époque Qing par les hommes aisés du fait de son ampleur majestueuse. À partir des années 1930, la variante féminine devient plus ajustée, pourvue de manches courtes et de fentes latérales. 22. Wayne Lee (née au Viêtnam en 1974) appartient à une famille d'immigrés chinois installés au Viêtnam et arrivés aux États-Unis avec les boat-people, en 1979. Elle fonde la marque Wayne en 2007 , à New York. 23. Le verbe "re-member" est mis entre guillemets par Lisa Lowe pour signifier à la fois "se remémore" et "re-compose" le passé. 24. Lisa Lowe, op. cit., p. 29.
} 


\section{Une combinatoire de mémoires empilées}

Quand les designers décrivent les matériaux de prospection qui servent de point de départ à leurs projets de collections, il arrive que la mémoire de l'architecture d'une veste unisexe chinoise ou du qipao des aînées de la famille se confonde avec celle du teeshirt. Dès ses premières collections, Lisa avait l'idée de se concentrer sur le teeshirt pour "retrouver la pureté du qipao avec ses lignes verticales qui ne découpent pas le corps en parties séparées". Aujourd'hui, elle explore les qualités du jersey et la structure minimaliste du tee-shirt qu'elle retourne, déconstruit ou transforme en petite robe du soir. Elle travaille parallèlement d'autres basiques américains aux formes simples comme le sweater, dont elle révolutionne les

Détail d'une blouse fluide en crêpe de soie, avec col officier et poches appliquées. Pièce de la collection Resort 2012 de 3.1 Phillip Lim, 2012. @ LEYLA BeLKAID

décoratives à la Chine, mais à une Chine imaginaire de film ou de roman. Si vous ne proposiez pas cela, c'est un peu comme si vous nétiez pas un vrai Chinois... alors pourquoi un journaliste se serait-il intéressé à votre collection? On ressentait cette pression, mais la situation a quand même changé depuis."

Ainsi, les sujets asiatico-américains "se confrontent à des attentes sociétales qui voudraient qu'ils soient ethniques à partir du moment où les autres pensent qu'ils devraient être plus proches de leurs racines ethniques que des racines américaines ${ }^{25}$ ". Pourtant, les réminiscences des patrimoines vestimentaires asiatiques et américains s'entrecroisent au fil de leurs narrations biographiques, à l'image des vêtements traditionnels qui cohabitent avec les jeans et les tenues de basket sur les étalages des marchés de Chinatown. proportions et les fonctions.

À l'instar de Phillip Lim ${ }^{26}$, pour plusieurs créateurs diasporiques, le concept du vêtement à bords droits parallèles enfilé par la tête constitue une base de travail neutre et stimulante. Wayne élabore "une certaine sorte de tee-shirts" aux détails sophistiqués, convaincue que ses clientes qui sont des femmes actives et pressées aimeraient "vivre dans un tee-shirt". Or cet archétype américain reste indissociable de l'univers des sportifs, des motards et de toute une série d'icônes populaires qui guident les pérégrinations imaginaires des sujets d'origine chinoise.

D'autres vêtements iconiques, comme les blousons en cuir et les jeans, affleurent des récits collectés à Manhattan. Objet de cohésion nationale, le pantalon en denim reste un symbole d'affranchissement et de liberté. Wayne s'en inspire pour créer des vêtements faciles (easy clothes), mais elle ne produit pas de jeans car leur conception requiert 
des compétences techniques spécifiques. Quant à Susan (pseudonyme), elle explore cet archétype avec l'espoir de parvenir un jour à développer un concept de pantalon qui ne soit pas un jean, mais qui en ait les qualités ergonomiques ${ }^{27}$. Elle rêve d'inventer "un pantalon qui se confonde avec nos gestes, notre quotidien et qui dure". Les attitudes corporelles associées au confort du pantalon traditionnel chinois se jumellent, selon sa perception de la dynamique du vêtement, avec celles induites par le port du jeans. L'assimilation des logiques vestimentaires inhérentes aux cultures des deux rives du Pacifique conduit dès lors à une construction du corps féminin qui privilégie l'aisance physique, la liberté de mouvement et une certaine forme d'androgynie.

La plupart des designers semblent conscients du caractère singulier de leur définition du vêtement comme culture matérielle susceptible de façonner l'espace d'un corps "différent", marqué par des pratiques corporelles et des traditions vestimentaires hybrides. Pour Thomas $\mathrm{Chen}^{28}$, l'écart majeur entre les conceptions orientales et occidentales du vêtement se situe au niveau de la perception du corps genré et de l'expression de la séduction par l'action du vêtement sur l'apparence corporelle. Ce paramètre permettrait, selon lui, d'élucider les spécificités du vocabulaire stylistique de ses collections. Il s'expliquerait par l'impact visuel de la chorégraphie quotidienne des corps en mouvement des personnes, proches ou anonymes, qui ont accompagné son enfance en Chine. Thomas postule que le style des produits qu'il met en circulation reste dominé par une approche plus orientale du corps puisqu'il ne suggère "rien d'ouvertement ou d'excessivement sexuel". Bien que les vêtements qui portent sa signature n'exercent pas de contraintes sur le corps et ne modifient pas ses formes naturelles, "le sens du corps y est toujours présent". L'analyse comparative du degré de voilement des différentes parties du corps par les artefacts étudiés montre que la tendance à valoriser le corps féminin sans mettre l'accent sur sa nudité et sans agir sur sa morphologie s'avère commune à la production de l'ensemble de mes interlocuteurs. À l'instar de Thomas, Lisa pressent la prégnance de la culture vestimentaire chinoise, mémorisée depuis l'enfance, sur l'écriture du corps habillé qu'elle développe à travers ses collections : "Je pense que les Asiatiques ont une vision différente du corps féminin... disons que leur sens du corps est en quelque sorte plus sublimé. C'est comme si plus nous voilons le corps, plus nous utilisons notre imagination pour conjurer le voilement, pour percevoir ce qu'est le corps... Je pense qu'il y a un sens du mystère qui ressort aussi de mes collections. Le corps est davantage présent métaphoriquement... pas dans le sens physique, mais métaphorique."

Les comportements vestimentaires associés à l'habillement domestique de l'enfant, de sa mère et de ses proches ont contribué à composer l'habitus du designer. "Histoire incorporée, faite nature, et par là oubliée en tant que telle, l'habitus est la présence agissante de tout le passé dont il est le produit ${ }^{29}$." Le style des collections d'artefacts conçues par les designers de mode sino-américains investigués cristallise leur perception des mondes passés et présents, ainsi que leur mémoire des expériences associées aux cultures chinoise et états-unienne et à la relation corps-vêtement induite par chacune. Le souvenir des techniques du corps et des attitudes vestimentaires des femmes de la communauté chinoise, aujourd'hui parvenues à un âge avancé, s'insinue dès lors dans la dynamique des vêtements qu'ils façonnent au fil des saisons. Bien que l'esthétique des tuniques, des vestes et des pantalons de ces femmes immigrées ne se confonde pas avec celle des propositions innovantes qui défilent sur les podiums de la Fashion Week new-yorkaise, la mémoire des performances incorporées aux conduites motrices et sensorielles de leurs corps habillés innerve les expressions contemporaines d'un style diasporique constitué à l'intersection d'appartenances plurielles. 